\title{
Numerical modelling on stabilizing large magnetic island by rf current
}

\section{for disruption avoidance}

\author{
Xiaojing Wang ${ }^{1,2}$, Qingquan $\mathrm{Yu}^{3, *}$, Xiaodong Zhang ${ }^{2,}$, Sizheng $\mathrm{Zhu}^{2}$, Xiaoguang $\mathrm{Wang}^{2}$, Bin $\mathrm{Wu}^{2}$ \\ ${ }^{1}$ University of Science and Technology of China, Hefei, China \\ ${ }^{2}$ Institute of Plasma Physics, Chinese Academy of Sciences, Hefei, China \\ ${ }^{3}$ Max-Planck-Institut für Plasmaphysik, D-85748, Garching, Germany
}

\begin{abstract}
Numerical modelling on tearing mode stabilization by rf current due to electron cyclotron current drive (ECCD) has been carried out for the purpose of disruption avoidance, focusing on stabilizing the magnetic island which can grow to a large width and therefore might cause plasma disruption. When the island has become large, a threshold in driven current for fully stabilizing the mode is found, and below this threshold the island width only slightly decreases. The island's O-point shifts radially towards the magnetic axis as the mode grows, so that applying ECCD at the minor radius of island's O-point has a stronger effect than that at the original equilibrium rational surface for stabilizing a large island. During the island growth the required driven current for mode stabilization increases with the island width, indicating that it is more effective to apply ECCD as early as possible for disruption avoidance, as observed in experiments. The numerical results have been compared with those obtained from the modified Rutherford equation.
\end{abstract}

Key words: disruption avoidance; tearing mode; magnetic island; rf current, electron cyclotron current drive; numerical modelling.

*E-mail: qiy@ipp.mpg.de and xdzhang@ipp.ac.cn 


\section{Introduction}

Plasma disruptions often occur in tokamak discharges, limiting the range of tokamak operational parameters such as the plasma beta value, current and density [1-3]. The fast decay of the plasma energy and current during disruptions can generate runaway electrons and cause huge thermal loads and mechanical stresses on the plasma facing components [1,3-8]. Disruption mitigation or avoidance is therefore a very important issue for a fusion reactor like ITER [4].

It is well known that low-m tearing modes (TMs) or neoclassical tearing modes (NTMs), especially the $m / n=2 / 1$ mode ( $m$ and $n$ are the poloidal and toroidal mode numbers), driven by unfavorable current profiles or perturbed bootstrap current [9-14], can possibly grow to a large amplitude and cause disruptions [15-19]. The stabilization of the 2/1 TMs has been shown to avoid disruptions in experiments $[3-5,16,17]$. Due to its good localization of power deposition, electron cyclotron wave (ECW) has been successfully used for avoiding disruptions on JFT-2M, TEXTOR, DIII-D, ASDEX Upgrade and FTU [3-5, 17, 18, 20-23]. Disruptions can happen due to the onset of large amplitude $m / n=2 / 1$ modes when the plasma density approaches the Greenwald limit [4, 5, $22,24]$. On JFT-2M tokamak, disruption avoidance was achieved reliably by injecting ECW around the $q=2$ surface [22], where $q$ is the safety factor. Continuous ECW has been shown to stabilize the 2/1 mode occupying 0.25 of the minor radius $a$ on TEXTOR [23]. On DIII-D, a saturated 2/1 island with a width $0.21 a$ was suppressed by a real-time feedback system which aligns ECW deposition to the $q=2$ surface $[3,21]$. Avoidance of disruptions was also observed by applying ECW at the $q=2$ surface on ASDEX Upgrade and FTU [4, 5, 17].

In addition to disruption avoidance, ECW has also been utilized for NTM stabilization in experiments [25-30]. Modulated current drive (MCD), which is phased with the O-point of a rotating magnetic island, is used to stabilize NTMs on ASDEX Upgrade, being more effective than a continuous or non-modulated current drive (NMCD) for a larger radial width of ECW deposition [2830]. Review papers on NTM stabilization are given in [31, 32]. There are already theoretical studies on the NTM stabilization by localized electron cyclotron current drive (ECCD), based on either the modified Rutherford equation (MRE) or numerical modelling of MHD equations [11, 12, 33-36]. However, to our knowledge no numerical modeling has been carried out yet to study the stabilization of large amplitude tearing modes driven by unfavorable plasma current density profiles, which are relevant for the disruptions, e.g., when the plasma density is close to the Greenwald limit [4, 5, 22, 24].

In the present paper, the stabilization of large amplitude $m / n=2 / 1$ tearing modes by rf current due to ECCD is studied numerically for the purpose of disruption avoidance, to investigate the important parameters affecting the mode stabilization for both MCD and NMCD. It is found that after nonlinear mode saturation, a threshold in driven current for fully stabilizing the mode exists. Below this threshold, the island width only slightly decreases. The O-point of a large island shifts away from the original equilibrium rational surface, so that it is necessary to trace its location in real-time for an accurate ECW deposition. When the driven current is applied during the island growth, the required driven current for mode stabilization increases with the island width, indicating that it is more effective to apply ECCD as early as possible when the island is still small, as observed in experiments.

In the next section, our theoretical model is introduced. The numerical results are presented in the third section. The discussion and summary are in the last section. 


\section{Calculation model}

Cylindrical geometry is utilized, and the magnetic field $\boldsymbol{B}$ is defined to be

$\boldsymbol{B}=B_{0 t} \boldsymbol{e}_{\boldsymbol{t}}-(n r / m R) B_{0 t} \boldsymbol{e}_{\boldsymbol{\theta}}+\nabla \psi \times \boldsymbol{e}_{\boldsymbol{t}}$,

where $\psi$ is the helical flux function, $m / r$ and $n / R$ are the wave vectors in $\boldsymbol{e}_{\boldsymbol{\theta}}$ (poloidal) and $\boldsymbol{e}_{\boldsymbol{t}}$ (toroidal) direction respectively, $R$ is major radius, and $B_{0 t}$ is the toroidal magnetic field.

The Ohm's law, the plasma vorticity equation and the plasma pressure evolution equation are utilized,

$$
\begin{aligned}
& \frac{\partial \psi}{\partial t}+\boldsymbol{v} \cdot \nabla \psi=E-\eta\left(j-j_{b}-j_{d}\right), \\
& \rho\left(\frac{\partial}{\partial t}+\boldsymbol{v} \cdot \nabla\right) \nabla^{2} \emptyset=\boldsymbol{e}_{\boldsymbol{t}} \cdot(\nabla \psi \times \nabla j)+\rho \mu \nabla^{4} \emptyset, \\
& \frac{3}{2}\left(\frac{\partial}{\partial t}+\boldsymbol{v} \cdot \nabla\right) p=\nabla \cdot\left(\chi_{\|} \nabla_{\|} p\right)+\nabla \cdot\left(\chi_{\perp} \nabla_{\perp} p\right)+Q,
\end{aligned}
$$

where $\boldsymbol{v}=\nabla \emptyset \times \boldsymbol{e}_{\boldsymbol{t}}, \emptyset$ is the stream function. $j=-\nabla^{2} \psi-2 n B_{0 t} /(m R), j_{b}=-c_{b} \frac{\sqrt{\varepsilon}}{B_{\theta}} \frac{\partial p}{\partial r}$ and $j_{d}$ are the plasma current density, the bootstrap current density, and the driven current density by ECW in the $\boldsymbol{e}_{\boldsymbol{t}}$ direction, respectively. $\rho$ is the plasma mass density, $\eta$ the plasma resistivity, $\mu$ the plasma viscosity, $p$ the plasma pressure, and $\nabla_{\|}$and $\nabla_{\perp}$ are the parallel and perpendicular gradient. $\chi_{\|}$and $\chi_{\perp}$ are the parallel and perpendicular heat transport coefficients, and $Q$ and $E$ are the heating power and the equilibrium electric field, respectively. $c_{b}$ is a constant of the order of unity, $\varepsilon=r / R$ the inverse aspect ratio, and $B_{\theta}$ the poloidal magnetic field. The plasma resistivity is calculated from $\eta=$ $E / j_{0}$, where $j_{0}$ is the equilibrium plasma current density. The plasma density and ion temperature are assumed to be constant for simplicity.

As the driven current density depends on both the wave deposition and the transport of the fast electrons produced by ECW, the fast electron density $n_{f}$ is calculated from [11]

$$
\frac{\partial n_{f}}{\partial t}=\nabla \cdot\left(\chi_{\| f} \nabla_{\|} n_{f}\right)+\nabla \cdot\left(\chi_{\perp f} \nabla_{\perp} n_{f}\right)+v_{f}\left(n_{f s}-n_{f}\right)
$$

where $\chi_{\| f}\left(\chi_{\perp f}\right)$ is the parallel (perpendicular) transport coefficient, $1 / v_{f}$ is the slowing-down time of the fast electrons, and $n_{f s}$ is the fast electron source density due to the EC waves $[11,36]$

$$
n_{f s}=n_{f s 0} \exp \left[-\left(\frac{r-r_{c d}}{w_{c d}}\right)^{2}\right] \Pi\left(h_{0}, \Delta h\right),
$$

where $n_{f s 0}$ and $r_{c d}$ are the amplitude and the radial depositing location of the source, respectively. $w_{c d}$ is the half width of the source. The square box function $\Pi\left(h_{0}, \Delta h\right)$ is equal to 1 for $\left|h-h_{0}\right|<$ $\Delta h$ and to 0 otherwise, defining the helical angle of the ECW deposition, where $h_{0}=\omega t, \omega$ is the relative rotation frequency between the mode and ECW deposition, and $\Delta h$ is the instantaneous wave deposition width along the helical angle $h=m \theta+n \xi$. In our computational model, the magnetic island does not rotate. The helical angle of the island's O-point is fixed at 0 , and the island's X-point at $\pi$ and $-\pi$. The ECW deposition rotates along the helical angle at a frequency $\omega$ with respect to the island. The helical angle of ECW deposition center, $h_{0}$, is calculated in each time step to compare with that of the island. Modulated ECCD is turned on only when $h_{0}$ varies in the half period from $-\pi / 2$ to $\pi / 2$. The driven current density by ECW is assumed to be proportional to the fast electron density. 
The radial profile of the original equilibrium safety factor $q$ utilized in our calculations is

$$
q(r)=q(0)\left[1+\left(\frac{r}{r o}\right)^{2 p}\right]^{\frac{1}{p}}
$$

where $q(0)$ is the safety factor at the magnetic axis, and ro determines the width of plasma current channel. $p=1, p=2$ and $p=4$ correspond to the so called peaked, rounded and flat current density profiles, respectively [15]. In agreement with early results [15], we find that the $m / n=2 / 1$ classical tearing mode can be unstable and grow to a large amplitude with the flat current density profile. This current profile has the features of a small current density amplitude outside the $q=2$ surface but a large radial gradient inside the $q=2$ surface, being similar to that in the density limit experiments caused by edge cooling. Therefore, the flat current density profile $(p=4)$ is utilized as the original equilibrium current density profile for our calculations. The local magnetic shear length at the $q=2$ rational surface $L_{q}=\left.\frac{q}{q^{\prime}}\right|_{r=r_{s 0}}=0.4 a$, and the bootstrap current is taken to be zero if not mentioned elsewhere, where the prime denotes the radial derivative, and $r_{s 0}=0.7 a$ is the radial location of the original equilibrium $q=2$ surface. In our model the effects of error field, toroidal mode coupling and shaping are neglected. If these effects are further taken into account, a sufficiently large island is expected to destabilize more islands and cause stochastic field and disruption. The studies presented in this paper is limited to the rf current stabilization of tearing mode instability which grows and saturates a large amplitude otherwise.

The simulation results are obtained by solving equations (2)-(4) simultaneously. The initial conditions include the original equilibrium q-profile defined in equation (7) and a small perturbation of the $m / n=2 / 1$ component helical flux. Once ECCD is applied, equations (5) and (6) are solved together with (2)-(4). The boundary conditions are $\left.f^{\prime}\right|_{r=0}=0$ and $\left.f\right|_{r=a}=0$ for all functions $f$ to be solved. In our calculations the time $t$ is normalized to $\tau_{R}$, the length to $a$, the helical flux to $a B_{0 t}$, the current density to $B_{0 t} / a$, and the transport coefficients to $a^{2} / \tau_{R}$, where $\tau_{R}=a^{2} \mu_{0} / \eta$ is the resistive time. The viscous time $\tau_{\mu}=a^{2} / \mu$ has been assumed to be ten times smaller than the resistive time.

\section{Numerical results}

Numerical results on the nonlinear growth and saturation of magnetic islands are first described in Section 3.1. The stabilization of saturated islands by ECCD is studied in Section 3.2, followed by the effect of radial ECW deposition location and other parameters on mode stabilization in Section 3.3. The stabilization of the island during its growth by ECCD is investigated in Section 3.4.

\subsection{Nonlinear growth and saturation of magnetic island}

By solving equations (2)-(4), the helical flux $\psi$, plasma current density and the poloidal field are calculated in each time step simultaneously. In figure 1 the time evolution of the normalized $\mathrm{m} / \mathrm{n}=$ 2/1 magnetic island width, $w / a$, and the radial location of the $q=2$ surface and of the island's Opoint are shown for $S=3.0 \times 10^{6}$, where $S=\tau_{R} / \tau_{A}$ is the Lundquist number, and $\tau_{A}$ is the Alfvén time. The adopted Lundquist number is a compromise for the sake of numerical tractability. The island grows in a time scale less than $0.01 \tau_{R}$ and then saturates at a large width $w=0.33 a$. As $\tau_{R} \propto$ $a^{2} T_{e}^{3 / 2}$, the value of $\tau_{R}$ depends on the plasma minor radius $a$ and the electron temperature $T_{e}$. For 
$a=0.5 \mathrm{~m}$ and $T_{e}=1 \mathrm{keV}$, one finds $\tau_{R}=11.2 \mathrm{~s}$. As mentioned in section 2 , the toroidal and shaping effects have not been taken into account in our calculations. These effects are expected to cause more islands and possibly field ergodicity and disruption once the $2 / 1$ island is sufficiently large. The qprofile, calculated by $q=r B_{0 t} /\left(R B_{\theta, 0 / 0}\right)$, evolves in time together with island growth, where $B_{\theta, 0 / 0}=-\partial \psi_{0 / 0} / \partial r$ is the $m / n=0 / 0$ component of the poloidal field. The $q=2$ surface moves inwards more than $0.03 a$ towards the magnetic axis in the time period $t / \tau_{R}=0-0.05$, since the plasma current density decreases inside the $q=2$ surface during the island growth as shown in the following figure 2, corresponding to an increase of the safety factor. The island's O-point moves even further inwards. The radial gradient of $\psi_{2 / 1}$ is negative around $r_{s}$ due to the low mode number and the equilibrium current density gradient. The inward drift of the island's O-point with respect to $r_{\mathrm{s}}$ is due to the negative radial gradient of $\psi_{2 / 1}$, which is proportional to the amplitude of $\psi_{2 / 1}$. If one neglects higher harmonic perturbations in the helical flux, the total helical flux is of the form $\psi(r, h)=\frac{q_{s}^{\prime} B_{\theta, 0 / 0}}{2 q_{s}}\left(r-r_{s}\right)^{2}-\psi_{2 / 1}(r) \cos (h)$, where $h$ is the helical angle. At the island's O-point ( $h=0$ ) the condition $\nabla \psi=0$ is satisfied, so that the O-point shifts inward with respect to $r_{\mathrm{s}}$, while the X-point (at $h=\pi$ ) has an outwards drift.

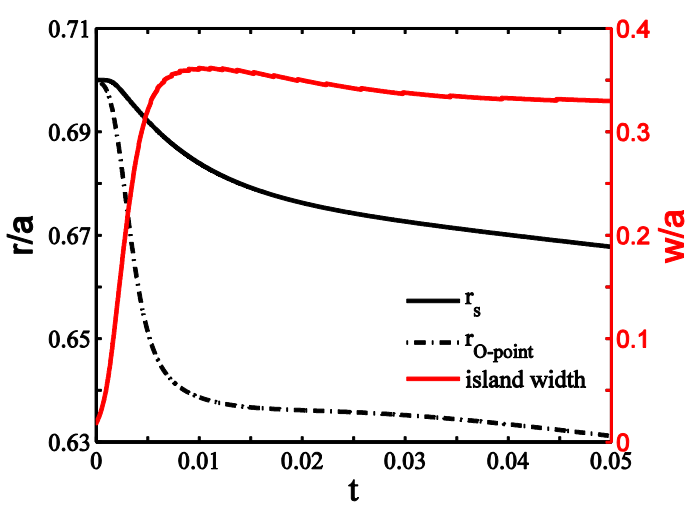

Figure 1. Time evolution of the normalized island width, $w / a$, and the radial location of the $q=2$ surface and of the island's O-point. The time $t$ is normalized to $\tau_{R}$.

The island width almost remains unchanged after $t / \tau_{R}=0.05$. However, the plasma current density profile still varies in a much longer time scale, especially in the central region from the magnetic axis to the island where plasma resistivity is smaller. Radial profiles of the $m / n=0 / 0$ component of the plasma current density $j$ are shown in figure 2 at $t / \tau_{R}=0.0,0.005,0.05$ and 0.3 . The vertical dashed lines show the corresponding radial locations of the inner and outer edges of the island at different times. The island moves inwards towards the magnetic axis during its growth. The change of the $m / n=0 / 0$ component plasma current density profile is due to the nonlinear term $\boldsymbol{v}$. $\nabla \psi$ in Ohm's law together with resistive current diffusion. For a large island in the Rutherford regime, the parallel gradient of the current density is nearly zero. The plasma resistivity does not change with time in our calculation. If the time evolution of the plasma resistivity is further taken into account, a larger change in the current density profile is expected due to the flattening in the electron temperature and resistivity profiles across the island. 


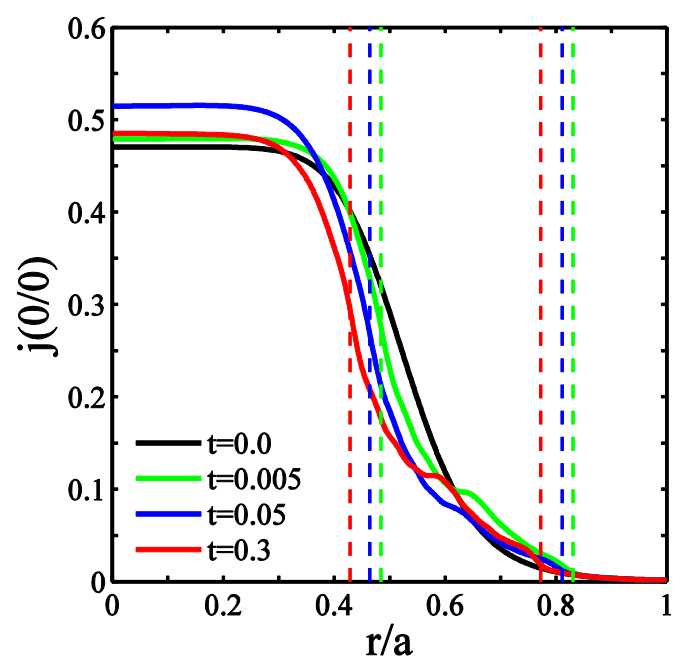

Figure 2. Radial profiles of the $m / n=0 / 0$ component plasma current density $j$ at $t / \tau_{R}=0.0$, $0.005,0.05$ and 0.3 . The vertical dashed lines show the corresponding radial locations of the inner and outer edges of the magnetic island at different times.

\subsection{Stabilizing magnetic island by ECCD after mode saturation}

After the nonlinear saturation in the island width, ECCD is applied at $t / \tau_{R}=0.05$ with the radial ECW deposition location aligned to the instant radius of the $q=2$ surface shown in figure 1, and other input parameters are $w_{c d} / a=0.05, \Delta \mathrm{h}=0.482, \omega=3 \times 10^{4} / \tau_{R}, v_{f}=3 \times 10^{3} / \tau_{R}, \chi_{\perp f}=$ $13.0 a^{2} / \tau_{R}$ and $\chi_{\| f} / \chi_{\perp f}=10^{8}$, if not mentioned elsewhere.

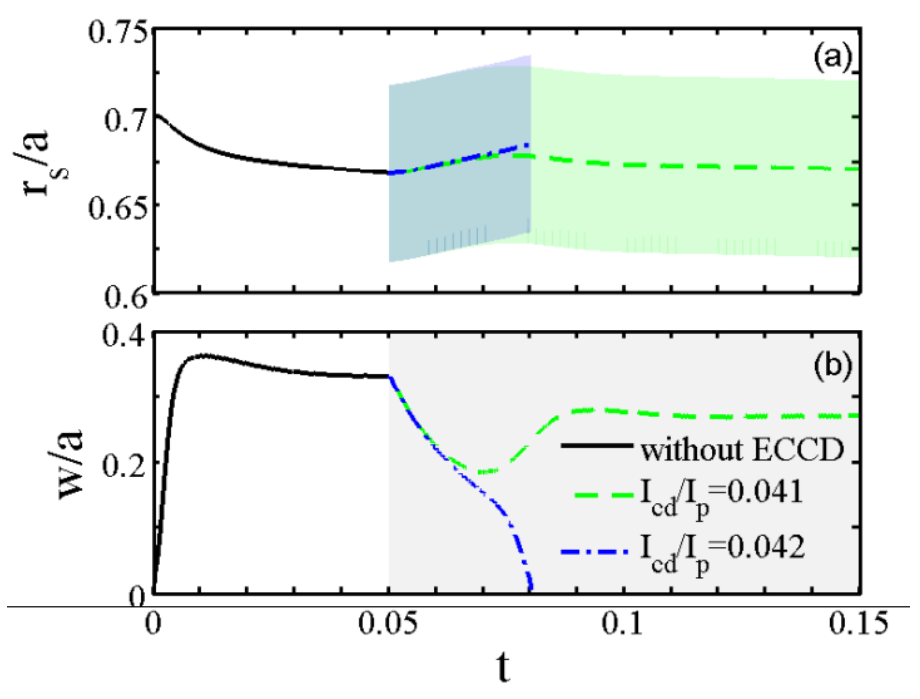

Figure 3. Time evolution of (a) the minor radius of the $q=2$ rational surface (the shadow region marks the radial-interval of MCD) and (b) island width (the shadow region marks the time interval of $\mathrm{MCD})$ for $I_{c d} / I_{p}=0.041(0.042)$.

The time evolution of (a) the minor radius of the $q=2$ surface and (b) the island width are shown 
in figure 3 for $I_{c d} / I_{p}=0.041(0.042)$, where $I_{c d}$ and $I_{p}$ are the driven current and plasma current, respectively. After the ECCD is turned on, the local current density increase due to both the rf current and the decreased island width, causing an outward movement of $r_{\mathrm{s}}$. For $I_{c d} / I_{p}=0.042$, the island is fully stabilized. For a slightly smaller driven current, $I_{c d} / I_{p}=0.041$, however, the island width first decreases but then still saturates at a large value. This is caused by the time evolution of the current density profile. The resistive diffusion time is much longer than the island growth/decay time, as shown in figure 2, causing a slow inward movement of $r_{\mathrm{s}}$ eventually for $I_{c d} / I_{p}=0.041$. It is seen that there is a threshold in the driven current for fully stabilizing the large island. Below this threshold the island width only slightly decreases. Similar results to those shown in figure 3 are also found for NMCD. It should be mentioned that for $I_{c d} / I_{p}=0.041$, the first decrease and then increase in island is due to the evolving plasma current profile, being different from the "phase flip", which is due to the change of the sign of the reconnected flux at the resonant surface when the island width is reduced to zero by ECCD, and then the island can bounce back with its old O-point becoming new X-point [37, 38]. For the case $I_{c d} / I_{p}=0.041$, the sign of the reconnected flux has not changed.

The saturated island width $w_{s a t}$ is shown in figure 4 as a function of $I_{c d} / I_{p}$ for different ECW deposition width $w_{c d}$, ranging from $0.01 a$ to $0.1 a$. There is a threshold in the driven current for fully stabilizing the mode in all cases. Below this threshold the island width only decreases a little. These results can also be explained in another way: there is a critical island width for mode stabilization by ECCD. Only when the island width is decreased to be below the critical width $(\sim 0.25 a$ in figure 4$)$ by a sufficiently large driven current, the island can then be fully stabilized. Experimental results also indicate an ECW power threshold for disruption avoidance [4]. The critical island width is similar to the marginal island width, $w_{\text {marg }}$, for the neoclassical tearing mode shown before [39]. When the island width is smaller than $w_{\text {marg }}$, the NTM decays due to the stabilizing effects of the finite perpendicular transport and the ion polarization current. As shown in the following figures 5 and 6 , however, the critical island width shown in figures 3 and 4 is due to the dependence of $\Delta_{0}^{\prime}$ and the stabilizing effect of the driven current on the island width. Comparing the results with different ECW deposition width, it is found that the thresholds in driven current for mode stabilization is significantly decreased when reducing $w_{c d}$ from $0.1 a$ to $0.05 a$. For $w_{c d}=0.01 a$, however, the decrease in the threshold is not scaled with $1 / w_{c d}$ when compared to the case with $w_{c d}=0.05 a$, indicating that a too small ECW deposition width will not greatly improve the stabilization of a large island. Figure 4 also shows that MCD is more effective than NMCD on mode stabilization for a large $w_{c d}$, as expected [12]. Similar results to figure 4 are also found with different equilibrium $q$-profiles. 


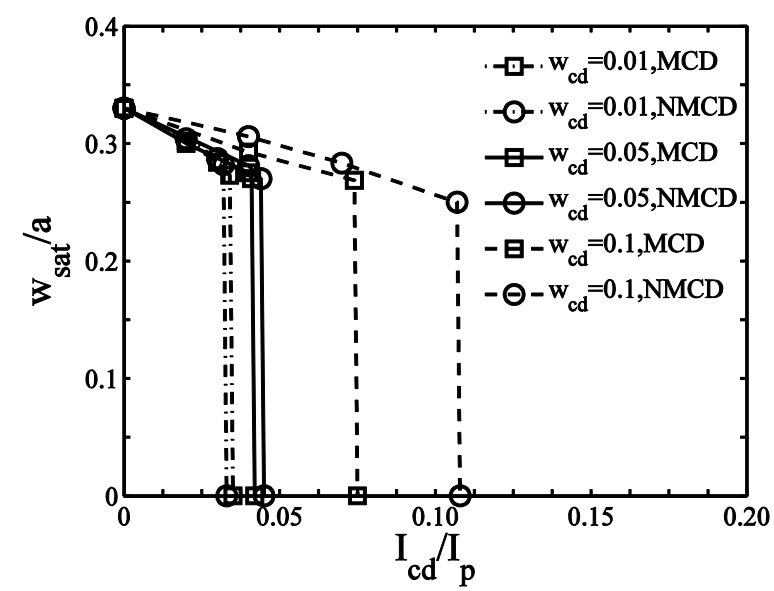

Figure 4. Saturated island width $w_{s a t}$ versus the driven current ratio $I_{c d} / I_{p}$ for different $w_{c d}$, ranging from $0.01 a$ to $0.1 a$.

The existence of a critical island shown in figures 3 and 4 can be understood from the modified Rutherford equation (MRE) [34, 36],

$$
\frac{\tau_{r}}{r_{s}} \frac{d w}{d t}=\Delta_{0}^{\prime} r_{s}+\Delta_{c d}^{\prime} r_{s}
$$

in which $\tau_{r}=\frac{\mu_{0} r_{s}^{2}}{1.22 \eta}, \Delta_{0}^{\prime}$ is the tearing mode stability index, and $\Delta_{c d}^{\prime}$ is the stabilizing term due to the driven current given by $[34,36]$

$$
\Delta_{c d}^{\prime} r_{s}=-\frac{16}{\pi} \frac{\mu_{0} I_{c d}}{s_{s} r_{s} B_{\theta}} \frac{r_{s}^{2}}{\left(2 w_{c d}\right)^{2}} \eta_{c d}
$$

where $s_{s}=\left.\frac{r q^{\prime}}{q}\right|_{r=r_{s}}$ is the local magnetic shear, and $\eta_{c d}$ is the so called stabilization efficiency. In figure $5 \eta_{c d}$ is shown as a function of the normalized island width $w / a$ for $w_{c d}=0.01 a$ (black curve), $0.05 a$ (red) and $0.1 a$ (blue). The solid and the dashed curves are for MCD and NMCD, respectively. The stabilizing efficiency increases with decreasing island width, and the increase is much more significant for MCD.

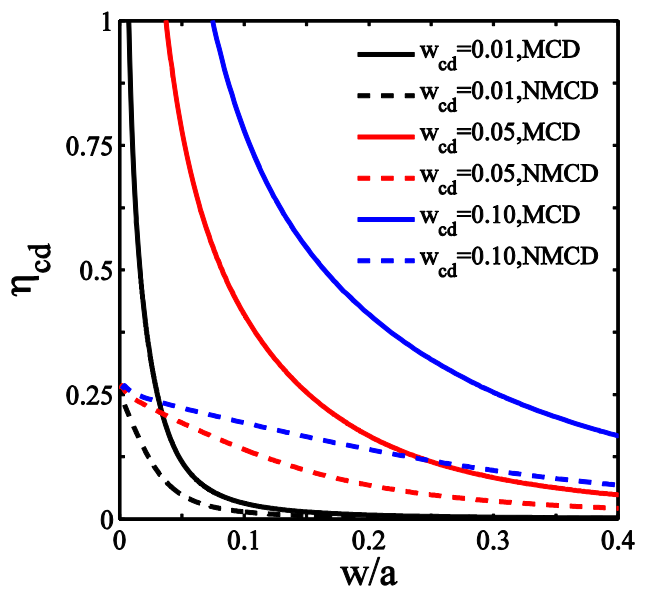

Figure 5. $\eta_{c d}$ versus the normalized island width $w / a$ for $w_{c d}=0.01 a$ (black curve), $0.05 a$ 
(red) and $0.1 a$ (blue). The solid and the dashed curves are for MCD and NMCD, respectively.
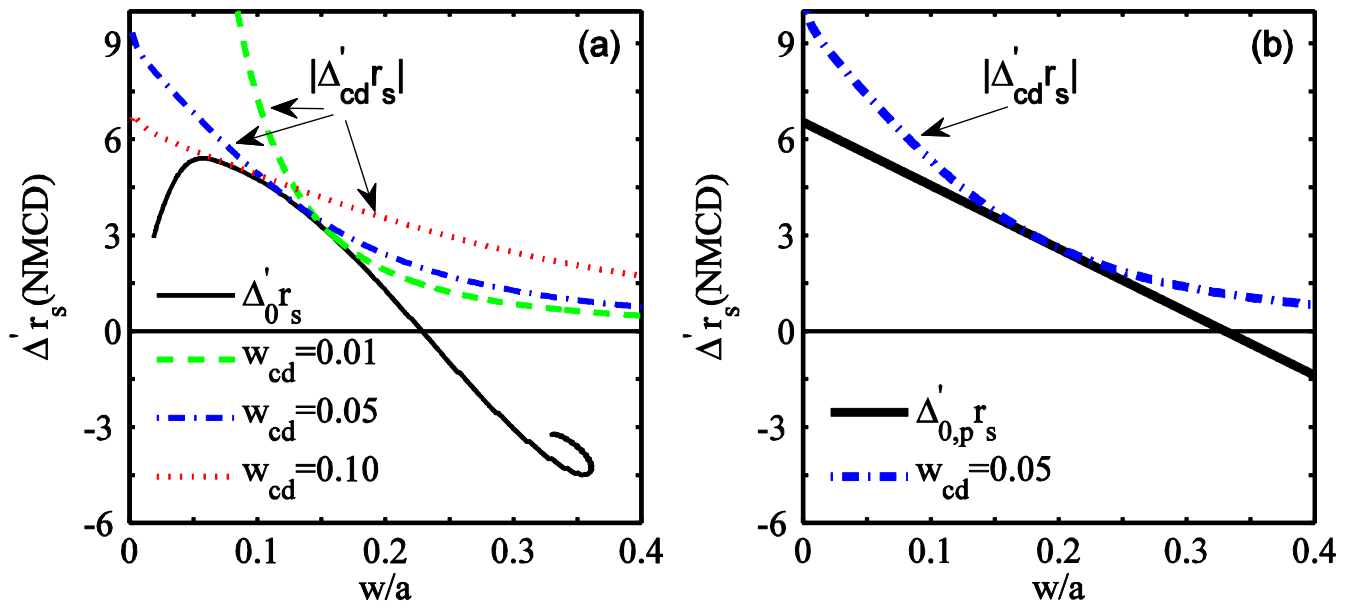

Figure 6. (a) $\Delta_{0}^{\prime} r_{s}$ and $\left|\Delta_{c d}^{\prime} r_{s}\right|$ versus the island width for NMCD. The solid curve shows the $\Delta_{0}^{\prime} r_{s}$ obtained numerically from equation (10). The dashed, dot-dashed and the dotted curves show the $\left|\Delta_{c d}^{\prime} r_{s}\right|$ for $w_{c d} / a=0.01\left(I_{c d} / I_{p}=0.022\right), 0.05\left(I_{c d} / I_{p}=0.038\right)$ and $0.1\left(I_{c d} / I_{p}=0.109\right)$. (b) A phenomenological form of $\Delta_{0}^{\prime}$ defined by Eq. (11), $\Delta_{0, p}^{\prime}$, is shown by the solid straight line, and the $\left|\Delta_{c d}^{\prime} r_{s}\right|$ for $w_{c d} / a=0.05\left(I_{c d} / I_{p}=0.041\right)$ is shown with the dot-dashed curve.

Corresponding to figure 1 , the tearing mode stability index $\Delta_{0}^{\prime} r_{s}$ is shown as a function of the island width in figure 6 (a) by the solid curve. The value of $\Delta_{0}^{\prime}$ is calculated by $[19,40]$

$$
\Delta_{0}^{\prime}=\frac{\psi_{1}^{\prime}\left(r_{+}\right)-\psi_{1}^{\prime}\left(r_{-}\right)}{\psi_{1}\left(r_{s}\right)}
$$

where $\psi_{1}$ is the perturbed helical flux function of the $m / n=2 / 1$ component, and $r_{+}$and $r_{-}$are the minor radius of the outer and the inner edges of the island obtained from numerical calculations. It is seen that $\Delta_{0}^{\prime}$ has a positive value for small island width as expected but a negative value at nonlinear mode saturation, indicating that it is inaccurate in determining the saturated island width. In figure 6(a) the values of $\Delta_{c d}^{\prime} r_{s}$ are also shown as a function of the island width for NMCD, with the dashed, dotdashed and the dotted curves for $w_{c d} / a=0.01\left(I_{c d} / I_{p}=0.022\right), 0.05\left(I_{c d} / I_{p}=0.038\right)$ and 0.1 $\left(I_{c d} / I_{p}=0.109\right)$, respectively. Increasing (decreasing) the value of $I_{c d} / I_{p}$, these curves for $\Delta_{c d}^{\prime} r_{s}$ simply shift upwards (downwards). The value of $I_{c d} / I_{p}$ in the figure 6(a) is selected for each $w_{c d} / a$ such that the $\Delta_{c d}^{\prime} r_{s}$ curve has only one cross point with the $\Delta_{0}^{\prime} r_{s}$ curve. Despite the inaccuracy of $\Delta_{0}^{\prime}$ in determining the saturated island width, it is still clear from figure 6(a) that once the island width is decreased to below a critical width by a sufficiently large driven current, $\left|\Delta_{c d}^{\prime}\right|$ will always be larger than $\Delta_{0}^{\prime}$ with further decrease in island width, so that the island will be fully stabilized as seen from numerical results in figures 3 and 4 . If the driven current is less than a critical value, the value of $\Delta_{0}^{\prime}$ is equal to or larger than that of $\left|\Delta_{c d}^{\prime}\right|$ at a certain island width, since the island width is still large, so that the island saturates at a large width.

Without the driven current, the value of $\Delta_{0}^{\prime}$ in MRE should be zero at nonlinear mode saturation. To satisfy this condition, a phenomenological form for $\Delta_{0}^{\prime}[41,42]$, 


$$
\Delta_{0, p}^{\prime}=\Delta_{0 \max }^{\prime}\left(1-w / w_{\text {sat }}\right),
$$

is assumed and shown in figure 6(b) with the solid straight line, where $\Delta_{0 \text { max }}^{\prime}$ is selected by requiring $\Delta_{0, p}^{\prime}$ passes through the maximum value of the $\Delta_{0}^{\prime}$ curve in figure $6(\mathrm{~b})$, and $w_{s a t}=0.33 a$ is the saturated island width. A case of $\left|\Delta_{c d}^{\prime} r_{s}\right|$ for $w_{c d} / a=0.05\left(I_{c d} / I_{p}=0.041\right)$ is shown with the dotdashed curve. The $\Delta_{0, p}^{\prime} r_{s}$ curve has only one cross point with the $\Delta_{c d}^{\prime} r_{s}$ curve at a larger island width than the $\Delta_{0}^{\prime}$ curve does.

The change of plasma current density profile after nonlinear mode saturation is also found to affect the mode stabilization. The saturated island width as a function of $I_{c d} / I_{p}$ is show in figure 7 with MCD applied at $t / \tau_{R}=0.05$ (solid curve) and 0.3 (dashed). The required driven current for fully stabilizing the mode is larger by applying rf current later at $t / \tau_{R}=0.3$, since the plasma current density profile at $t / \tau_{R}=0.3$ is different from that at $t / \tau_{R}=0.05$ as shown in figure 2 .

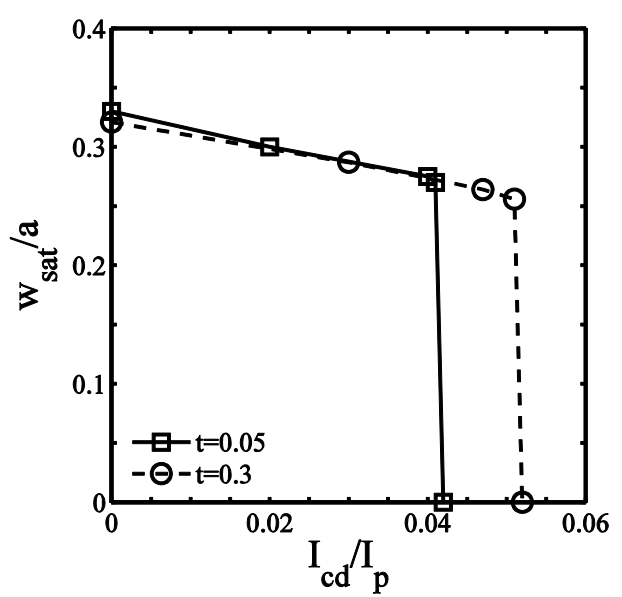

Figure 7. Saturated island width versus $I_{c d} / I_{p}$ for applying MCD at the time $t / \tau_{R}=0.05$ (solid) and 0.3 (dashed).

To examine the effect of the $m / n=0 / 0$ and $2 / 1$ components driven current on mode stabilization, in figure 8 the saturated island width is shown as a function of $I_{c d} / I_{p}$ for MCD. The solid curve is obtained by including the full components of the driven current, while for the dashed and the dot-dashed curves, the $m / n=0 / 0$ and $2 / 1$ component driven current are taken be zero in calculations, respectively. Without the $2 / 1$ component of the driven current, the required rf current for mode stabilization is significantly larger, as expected. When the $m / n=0 / 0$ component driven current is taken to be zero, the required rf current is about two times larger than that with full driven current, indicating that the change of plasma current density profile by ECCD significantly affects the mode stabilization. 


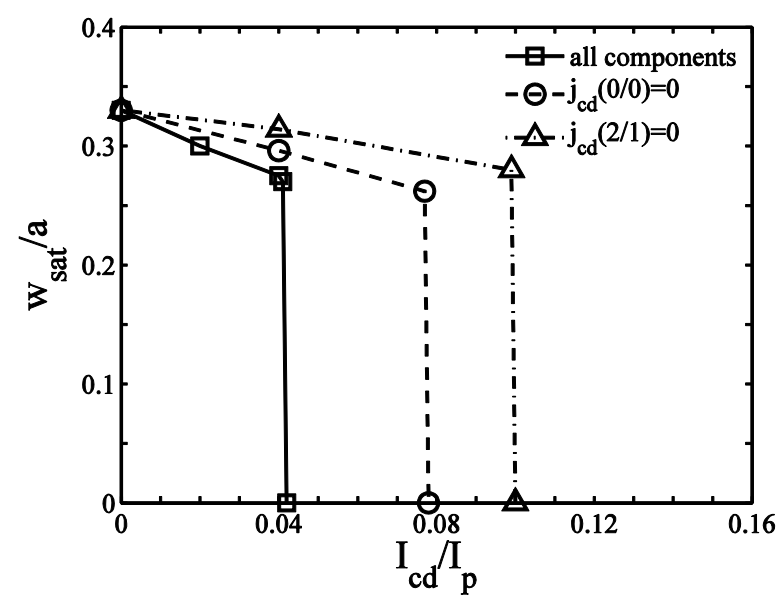

Figure 8. Saturated island width versus $I_{c d} / I_{p}$ for MCD with different components of driven current applied.

\subsection{Effects of ECW deposition location and other parameters}

There is usually a certain level of inaccuracy in measuring the location of the rational surface in experiments. Hence, the effect of the radial ECW deposition location on mode stabilization is studied. In figure 9 the time evolution of the island width for different deposition locations of the modulated ECCD are shown, with the time-interval in which MCD is applied shaded by grey. The thick solid curve is the case without ECCD. The thin dashed, dot-dashed, dotted, and the solid curves are obtained with $I_{c d} / I_{p}=0.045$ for $x=-0.01,0.0,0.01$, and 0.02 , respectively, where $x=\left(r_{c d}-r_{s}\right) / a$. The case for $x=-0.02$ is not shown, which is located between the two curves with $x=-0.01$ and $x=0.0$. The efficiency of ECCD deposited at the outer side of rational surface is lower than that the inner side. Applying ECCD at $x=-0.01$ is slightly better than that at $x=0.0$, since the island's O-point is shifted inwards towards the magnetic axis.

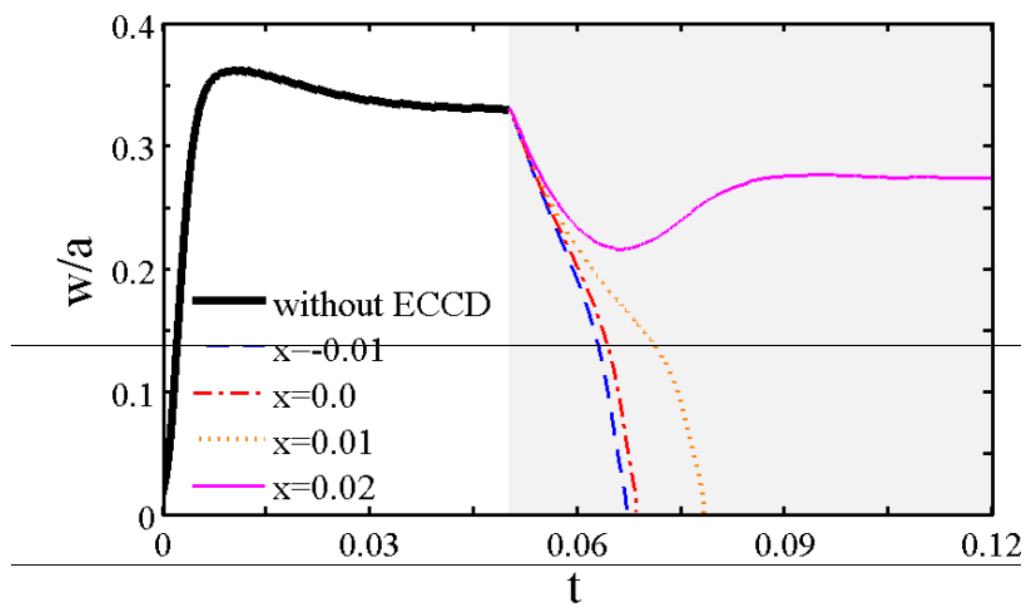

Figure 9. Time evolution of the island width for different radial ECW deposition position, with $x=$ $\left(r_{c d}-r_{s}\right) / a$ ranging from -0.01 to 0.02 . The time-interval in which MCD is applied is shaded by grey. 
For figures 3-8 ECCD is deposited at the real-time location of the rational surface in calculations. As shown in figure 1, the rational surface moves inwards more than $0.03 a$ from its original location during the mode growth. The difference between the ECW deposition at the instant rational surface $r_{s}$ (solid curve) and at the initial rational surface $r_{s 0}$ (dashed) is compared in figure 10(a), in which the saturated island width is shown as a function of $I_{c d} / I_{p}$. The square (circle) symbols are for MCD (NMCD). The required driven current for stabilizing the mode is $22 \%$ larger for ECW deposition at the original $q=2$ surface than that at the instant $q=2$ surface.
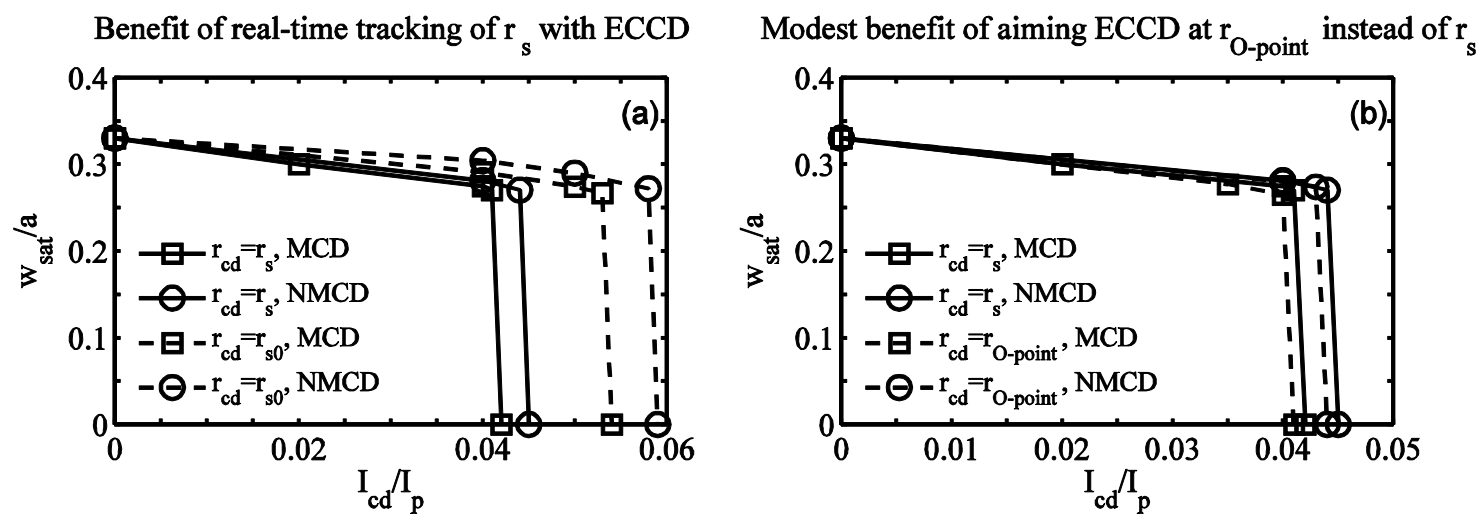

Benefit of high $v_{\mathrm{f}} / \omega$ for $\operatorname{MCD}\left(v_{\mathrm{f}}=3 \times 10^{3} / \tau_{\mathrm{R}}\right)$
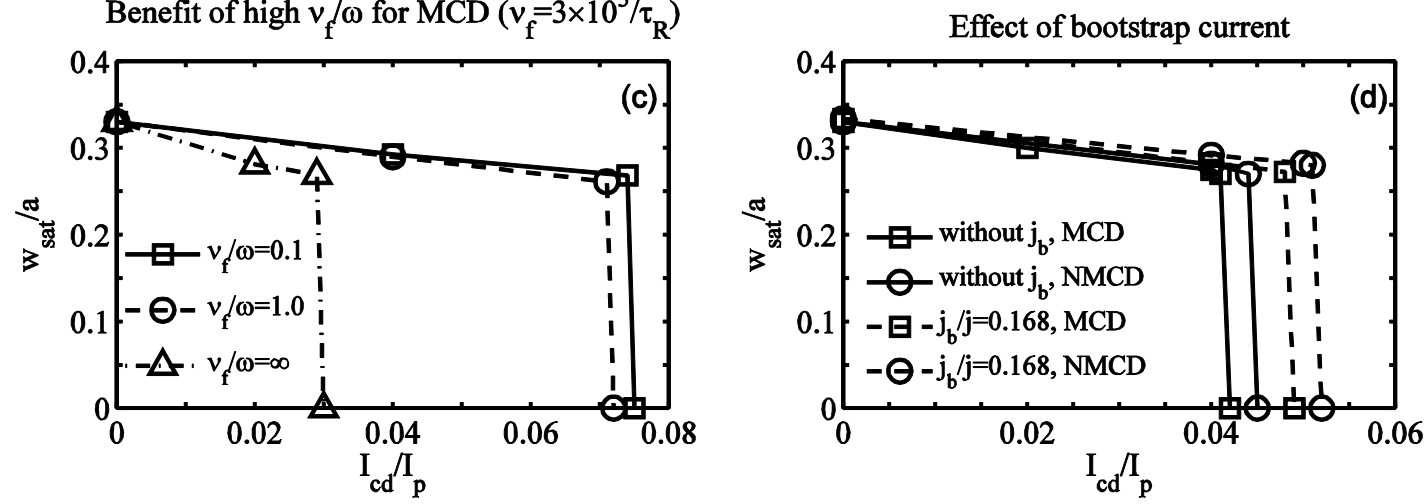

Figure 10. Saturated island width versus $I_{c d} / I_{p}$, for (a) ECW deposition at the instant rational surface $r_{s}$ (solid curve) and at the initial rational surface $r_{s 0}$ (dashed), (b) ECW deposition at $r_{s}$ (solid) and at the radial location of island's O-point $r_{\mathrm{O} \text {-point }}$ (dashed), (c) different $v_{f} / \omega$ for MCD with $v_{f}=3 \times 10^{3} / \tau_{R}$ and (d) different bootstrap current density fraction.

The island's O-point is shifted away from the rational surface during the island growth, as shown in figure 1. To compare the two cases with the ECW deposition at the instant rational surface $r_{S}$ (solid curve) and at the radial location of island's O-point $r_{\mathrm{O} \text {-point }}$ (dashed), in figure $10(\mathrm{~b})$ the saturated island width is shown as a function of $I_{c d} / I_{p}$ for these two cases. The square (circle) symbols are for MCD (NMCD). It is seen that ECCD aligned to the radial location of island's O-point is slightly better than that to the real-time rational surface. After ECCD is turned on, the island's O-point approaches the rational surface as the island width decreases.

Other parameters affecting the mode stabilization are also investigated. The saturated island width as a function of $I_{c d} / I_{p}$ is shown in figure 10(c) for different island rotation frequency $\omega$ with MCD 
and $w_{c d}=0.1 a$, where the solid, the dashed and the dot-dashed curves are for $v_{f} / \omega=0.1(\omega=3 \times$ $\left.10^{4} / \tau_{R}\right), \quad v_{f} / \omega=1\left(\omega=3 \times 10^{3} / \tau_{R}\right)$ and $v_{f} / \omega=\infty(\omega=0)$, respectively. A large $v_{f} / \omega$ corresponds to a stronger stabilizing effect. This effect is significant only when the island rotation period $1 / \omega$ is longer than the driven current rising time $1 / v_{f}$. For a smaller $\omega$ (smaller than the fast electron collision frequency $v_{f}$ ), the rf current has more time to rise to a larger amplitude in the half period when the rf current is turned on for MCD, therefore leading to a lager stabilizing effect. While for the case with a larger $\omega$ (much larger than $v_{f}$ ), the rf current amplitude for MCD only rises to half the value of the continuous current drive. In the limit when $\omega$ approaches zero, the MCD has the same driven current amplitude as the continuous current drive, although the rf current is only deposited around the O-point.

For above results the bootstrap current is assumed to be zero. Including the bootstrap current in our calculations, the saturated island width increases little even when the local bootstrap current density fraction at the $q=2$ surface, $j_{b} / j$, is increased to $22 \%$, indicating that the bootstrap current perturbation is not important in affecting the island width at saturation if $j_{b}$ is not too large. The saturated island width is shown in figure $10(\mathrm{~d})$ as a function of $I_{c d} / I_{p}$ with $\chi_{\perp}=13.0 \mathrm{a}^{2} / \tau_{R}$ and $\chi_{\|} / \chi_{\perp}=10^{8}$ for $j_{b} / \mathrm{j}=0.0$ (solid curve) and 0.168 (dashed), and the square (circle) symbols are for MCD (NMCD). The required driven current for mode stabilization increases a little when including the bootstrap current, to compensate the missing bootstrap current inside the island.

\subsection{Effect of the initial magnetic island width when applying ECCD}

As expected from the dependence of $\Delta_{c d}^{\prime}$ on the island width shown in figure 6 , it should be more effective to stabilize the $2 / 1$ mode by ECCD when the island width is small. The time evolution of the island width is shown in figure 11 with zero bootstrap current for different initial island widths $w_{0}$, ranging from $0.018 a$ to $0.071 a$. MCD is turned on at the time $t / \tau_{R}=0$ with $I_{c d} / I_{p}=0.03$ and $w_{c d}=0.05 a$. It is seen that the island is stabilized for a small $w_{0}$ but still grows for a sufficiently large one. For the case with $w_{0}=0.036 a$ the island is stabilized in a longer time scale. Figure 11 confirms that for a give driven current amplitude, ECCD has a larger stabilization effect for a smaller island width. The original equilibrium q-profile utilized is unstable to the tearing mode, so that the mode still grows for a larger initial island width because of the weaker stabilizing effect of ECCD in this case. 


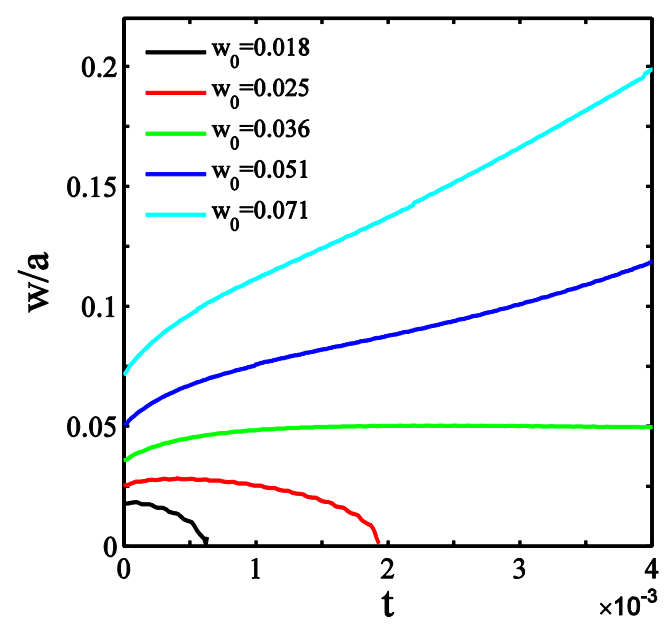

Figure 11. Time evolution of the island width for different initial widths $w_{0}$. MCD is turned on at the time $t / \tau_{R}=0$ with $I_{c d} / I_{p}=0.03$.

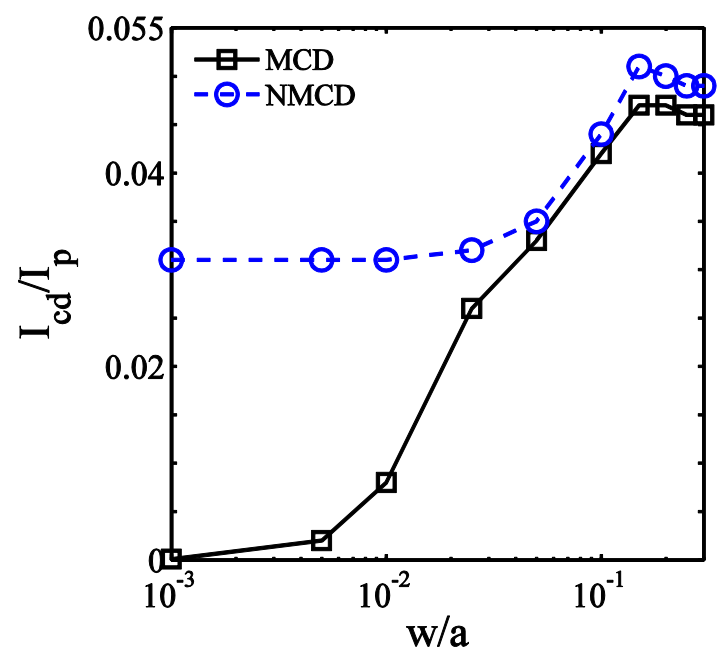

Figure 12. Required $I_{c d} / I_{p}$ for mode stabilization versus the normalized island width for MCD (solid curve) and NMCD (dashed).

The required $I_{c d} / I_{p}$ for fully stabilizing the $2 / 1$ mode is shown as a function of the island width in figure 12, in which ECCD is applied when the island width grows to the value shown by the horizontal axis. The solid (dashed) curve is for MCD (NMCD). It is seen that before nonlinear mode saturation, the required driven current for mode stabilization increases with $w$, indicating the advantage of applying ECCD earlier. For a small island with $w \sim 0.01 a$ or smaller, MCD is much more effective than NMCD for stabilizing the mode. The existence of the maximum driven current at $w / a=0.15$ is due to the change of current density profiles during mode evolution. Experiment results of DIII-D have shown that, by applying ECCD earlier, complete avoidance of the $3 / 2$ NTM onset was achieved at a higher $\beta_{N}$ [43]. The onset of a $2 / 1$ mode in the presence of a $3 / 2$ mode was completely avoided by preemptively applying ECCD at $q=2$ surface [21]. On JT-60U, the excitation of the $3 / 2$ NTM was significantly postponed when ECW was injected before the appearance of the mode [44]. 
Our numerical results agree with experimental findings.

\section{Discussion and summary}

In order to compare our numerical results to those obtained from the MRE, the required driven current for mode stabilization is shown as a function of $w_{c d} / a$ in figure 13. The solid curves are obtained from numerical calculations. The square (circle) symbols are for MCD (NMCD). ECCD is turned on at $t / \tau_{R}=0.05$ after mode saturation. The dashed curves are obtained from the MRE using the $\Delta_{0}^{\prime}$ calculated by equation (10), and the value of $I_{c d} / I_{p}$ is found for each $w_{c d} / a$ when the $\Delta_{c d}^{\prime} r_{s}$ curve has one cross point with the $\Delta_{0}^{\prime} r_{s}$ curve, as shown in figure 6(a). It is seen that numerical results have the same trend as those from MRE. As the values of $\Delta_{0}^{\prime} r_{s}$ shown in figure 6(a) are inaccurate for describing the effect of plasma current density profile on the nonlinear evolution of a large island, there is a quantitative difference between numerical results and those from MRE. In standard Rutherford equation the island width has to be assumed sufficiently small such that the boundary layer theory, basing on the match between the outer and inner region solutions, is valid. In our numerical calculations, the change of the plasma current density profile due to ECCD and island evolution is selfconsistently calculated, and $\Delta_{0}^{\prime} r_{s}$ is not needed in determining the island width. The MRE results using the phenomenological form for $\Delta_{0}^{\prime}, \Delta_{0, p}^{\prime}$, are also shown in figure 13 by dot-dashed curves, being closer to numerical results.

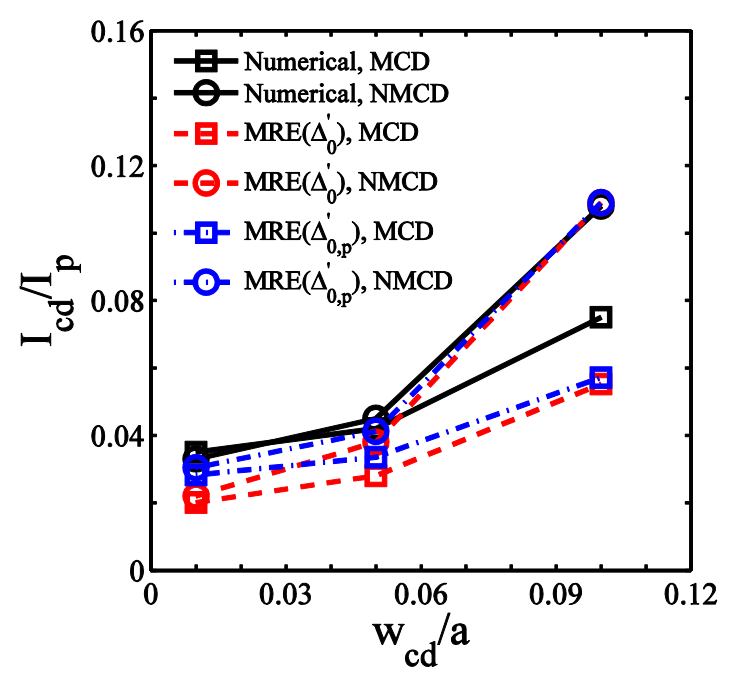

Figure 13. Required $I_{c d} / I_{p}$ for mode stabilization versus $w_{c d} / a$, obtained numerically with ECCD turned on at $t / \tau_{R}=0.05$ after mode saturation (solid curves). The dashed (dot-dashed) curve is obtained from MRE using the $\Delta_{0}^{\prime}\left(\Delta_{0, p}^{\prime}\right)$. The square (circle) symbols are for MCD (NMCD).

Our results show that the required driven current for mode stabilization is small when the island is small, and it increases with the island width during the mode growth as shown in figure 12, indicating that it is more effective to apply ECCD as early as possible, in agreement with experimental observation [21, 43, 44]. MCD is much more effective than NMCD for stabilizing a small island. For this purpose, early detection of the $m / n=2 / 1$ island to identify both its radial location and phase is required in discharges, such that less driven current is required for mode stabilization. If always 
applying NMCD at the $q=2$ surface to prevent the $2 / 1$ mode growth, the required driven current is about a few percent of the plasma current, depending on the plasma parameters.

After nonlinear mode saturation, a sufficiently large driven current is required to exceed a threshold for mode stabilization, as shown in figure 4 . When the driven current is below the threshold, there is only a weak stabilizing effect. The rational surface shifts away from its original location for a large island. The required driven current for mode stabilization is decreased by about twenty percent when ECCD is deposited at the real-time $q=2$ rational surface, compared to that at the original one. The mode stabilization by MCD is more effective when the island rotation period is longer than the driven current rising time. This condition can be realized if a slowly rotating resonant magnetic perturbation is externally applied to control the island rotation frequency [13].

In some experiments, localized ECW heating rather than ECCD is utilized for mode stabilization. The localized heating changes the local plasma resistivity and therefore the current density, having a similar mechanism as localized ECCD. Numerical calculations about the effect of localized ECW heating on mode stabilization are still required.

In summary, the stabilization of the $m / n=2 / 1$ classical tearing modes by ECCD for disruption avoidance is investigated numerically. It is found that:

(1) After nonlinear mode saturation, a threshold in driven current exists for stabilizing a large island, and below the threshold the island width only slightly decreases. ECCD deposition at the island's O-point has almost the same effect as that at the real-time rational surface but is more effective than that at the original rational surface.

(2) The required driven current for fully stabilizing the mode linearly increases with the island width during island growth, indicating that it is more efficient for mode stabilization to apply ECCD as early as possible. MCD is much more effective than NMCD for stabilizing a small island.

(3) The numerical results have the same trend as those obtained from the modified Rutherford equation. Due to the uncertainty in the values of the tearing mode stability index for a large island, there is a quantitative difference between them.

\section{Acknowledgement}

This work was supported by the National Magnetic Confinement Fusion Science Program of China (Contract No. 2012GB103000 and 2012GB103003) and the National Natural Science Foundation of China (Grant No. 11475225). Numerical computations were performed on the ShenMa High Performance Computing Cluster at the Institute of Plasma Physics, Chinese Academy of Sciences. 


\section{References}

[1] Park W. et al 1995 Phys. Rev. Lett. 751763

[2] Waddell B. V. et al 1978 Phys. Rev. Lett. 411386

[3] Luce T. C. et al 2005 J. Phys.: Conf. Ser. 25252

[4] Esposito B. et al 2011 Plasma Phys. Control. Fusion 53124035

[5] Esposito B. et al 2009 Nucl. Fusion 49065014

[6] Boozer A. H. 2012 Phys. Plasmas 19058101

[7] Lehnen M. et al 2013 Nucl. Fusion 53093007

[8] Riccardo V. and JET EFDA Contributors 2003 Plasma Phys. Control. Fusion 45 A269

[9] Westerhof E. 1987 Nucl. Fusion 271929

[10] Westerhof E. 1990 Nucl. Fusion 301143

[11] Yu Q. et al 2000 Phys. Plasmas 7312

[12] Yu Q. et al 2004 Phys. Plasmas 111960

[13] Yu Q. and Günter S. 2008 Nucl. Fusion 48065004

[14] Perkins F. W. et al, in Proceedings of the 24th EPS Conference on Controlled Fusion and Plasma Physics (European Physical Society), 9-13 June 1997, Berchtesgaden, Germany, Vol. 3, p. 1017

[15] White R. B. et al 1977 Phys. Rev. Lett. 391618

[16] Holmes J. A. et al 1979 Nucl. Fusion 191333

[17] Esposito B. et al 2011 Nucl. Fusion 51083051

[18] Petty C. C. et al 2004 Nucl. Fusion 44243

[19] White R. B. 1986 Rev. Mod. Phys. 58183

[20] Maraschek M. et al 2005 Nucl. Fusion 451369

[21] Prater R. et al 2007 Nucl. Fusion 47371

[22] Hoshino K. et al 1992 Phys. Rev. Lett. 692208

[23] Classen I. G. J. et al 2007 Phys. Rev. Lett. 98035001

[24] Greenwald M. 2002 Plasma Phys. Control. Fusion 44 R27

[25] La Haye R. J. et al 2002 Phys. Plasmas 92051

[26] La Haye R. J. et al 2006 Nucl. Fusion 46451

[27] Felici F. et al 2012 Nucl. Fusion 52074001

[28] Gantenbein G. et al 2000 Phys. Rev. Lett. 851242

[29] Zohm H. et al 2001 Phys. Plasmas 82009

[30] Zohm H. et al 2001 Nucl. Fusion 41197

[31] La Haye R. J. 2006 Phys. Plasmas 13055501

[32] Maraschek M. 2012 Nucl. Fusion 52074007

[33] De Lazzari D. and Westerhof E. 2009 Nucl. Fusion 49075002

[34] Hegna C. C. and Callen J. D. 1997 Phys. Plasmas 42940

[35] Sauter O. 2004 Phys. Plasmas 114808

[36] Giruzzi G. et al 1999 Nucl. Fusion 39107

[37] Lazzaro E. and Coelho R. 2002 The European Physical Journal D-Atomic, Molecular, Optical and Plasma Physics 1997

[38] Borgogno D. et al 2014 Phys. Plasmas 21060704

[39] Sauter O. et al 2002 Plasma Phys. Control. Fusion 441999

[40] Furth H. P. et al 1963 Phys. Fluids 6459 
[41] Wesson J., Tokamaks, 3rd ed. (Oxford University Press), 2004

[42] White R. B. et al 1977 Phys. Fluids 20800

[43] La Haye R. J. et al 2005 Nucl. Fusion 45 L37

[44] Nagasaki K. et al 2003 Nucl. Fusion 43 L7 